\title{
SHARP CAPACITY ESTIMATES IN S-JOHN DOMAINS
}

\author{
CHANG-YU GUO
}

\begin{abstract}
It is well-known that several problems related to analysis on $s$-John domains can be unified by certain capacity lower estimates. In this paper, we obtain general lower bounds of $p$ capacity of a compact set $E$ and the central Whitney cube $Q_{0}$ in terms of the Hausdorff $q$-content of $E$ in an $s$-John domain $\Omega$. Moreover, we construct several examples to show the essential sharpness of our estimates.
\end{abstract}

\section{INTRODUCTION}

Recall that a bounded domain $\Omega \subset \mathbb{R}^{n}$ is a John domain if there is a constant $C$ and a point $x_{0} \in \Omega$ so that, for each $x \in \Omega$, one can find a rectifiable curve $\gamma:[0,1] \rightarrow \Omega$ with $\gamma(0)=x, \gamma(1)=x_{0}$ and with

$$
C d(\gamma(t), \partial \Omega) \geq l(\gamma([0, t]))
$$

for each $0<t \leq 1$. F. John used this condition in his work on elasticity [13] and the term was coined by Martio and Sarvas [18]. Smith and Stegenga [21] introduced the more general concept of $s$-John domains, $s \geq 1$, by replacing (1.1) with

$$
C d(\gamma(t), \partial \Omega) \geq l(\gamma([0, t]))^{s} .
$$

The condition 1.1 is called a "twisted cone condition" in literature. Thus condition 1.2 should be called a "twisted cusp condition".

In the last twenty years, $s$-John domains has been extensively studied in connection with Sobolev type inequalities; see [3, 11, 9, 14, 17, 21]. In particular, Buckley and Koskela [3] have shown that a simply connected planar domain which supports a Sobolev-Poincaré inequality is an $s$ John domain for an appropriate $s$. Smith and Stegenga have shown that an $s$-John domain $\Omega$ is a $p$-Poincaré domain, provided $s<\frac{n}{n-1}+\frac{p-1}{n}$. In particular, if $s<\frac{n}{n-1}$, then $\Omega$ is a $p$-Poincaré domain for all $1 \leq p<\infty$. These results were further generalized to the case of $(q, p)$-Poincaré domains in [11, 14, 17]. Recall that a bounded domain $\Omega \subset \mathbb{R}^{n}, n \geq 2$, is said to be a $(q, p)$-Poincaré domain if there exists a constant $C_{q, p}=$

2010 Mathematics Subject Classification. 30C65,46E35.

Key words and phrases. s-John domain, Hausdorff $q$-content, $p$-Capacity.

C.Y.Guo was partially supported by the Academy of Finland grant 131477 and the Magnus Ehrnrooth foundation. 
$C_{q, p}(\Omega)$ such that

$$
\left(\int_{\Omega}\left|u(x)-u_{\Omega}\right|^{q} d x\right)^{1 / q} \leq C_{q, p}\left(\int_{\Omega}|\nabla u(x)|^{p} d x\right)^{1 / p}
$$

for all $u \in C^{\infty}(\Omega)$. Here $u_{\Omega}=f_{\Omega} u(x) d x$. When $q=p, \Omega$ is termed a $p$-Poincaré domain and when $q>p$ we say that $\Omega$ supports a SobolevPoincaré inequality.

The recent studies [1, 5, 7] on mappings of finite distortion have generated new interest in the class of $s$-John domains. In particular, uniform continuity of quasiconformal mappings onto $s$-John domains was studied in [4, 6].

The proofs for both types of problems rely on certain capacity estimates for subsets of $s$-John domains. To be more precise, for the problem related to Sobolev-Poincaré inequalities, one uses the idea of Maz'ya [19, 20] to reduce the problem to capacity estimates of the form

$$
\operatorname{Cap}_{p}\left(E, Q_{0}, \Omega\right) \geq \psi(|E|),
$$

where $Q_{0}$ is the fixed Whitney cube containing the (John) center $x_{0}$ and $E$ is an admissible subset of $\Omega$ disjoint from $Q_{0}$; for (1.3), $\psi(t)=C t^{p / q}$, see also [8, 17]. Here, by admissible we mean that $E$ is an open set so that $\partial E \cap \Omega$ is a smooth submanifold. As for the uniform continuity of quasiconformal mappings onto $s$-John domains, one essentially needs a capacity estimate of the form

$$
\operatorname{Cap}_{n}\left(E, Q_{0}, \Omega\right) \geq \psi(\operatorname{diam} E),
$$

where $E$ is a continuum in $\Omega$ disjoint from the central Whitney cube $Q_{0}$; see [4. Thus one could expect that a more general capacity estimate of the form

$$
\operatorname{Cap}_{p}\left(E, Q_{0}, \Omega\right) \geq \psi\left(\mathcal{H}_{\infty}^{q}(E)\right)
$$

holds in certain $s$-John domains $\Omega$, where $E$ is a compact set in $\Omega$ disjoint from the central Whitney cube $Q_{0}$ and $\mathcal{H}_{\infty}^{q}(E)$ is the Hausdorff $q$-content of $E$. We confirm this expectation by showing the following result.

Theorem 1.1. Let $\Omega \subset \mathbb{R}^{n}, n \geq 2$, be an $s$-John domain. For $0<$ $\varepsilon<1,1 \leq p \leq n$ and $q \geq s(n-1)+1-p+\varepsilon$, there exists a positive constant $C(n, p, q, s, \varepsilon)$ such that

$$
\operatorname{Cap}_{p}\left(E, Q_{0}, \Omega\right) \geq C(n, p, q, s, \varepsilon)\left(\mathcal{H}_{\infty}^{q}(E)\right)^{\frac{s(n-1)+1-p+\varepsilon}{q}},
$$

whenever $E \subset \Omega$ is a compact set disjoint from $Q_{0}$.

Remark 1.2. If $p=n, q=1$ and $E \subset \Omega$ is a continuum, then (1.5) reduces to the estimate

$$
\operatorname{Cap}_{n}\left(E, Q_{0}, \Omega\right) \geq C(n, s, \varepsilon)(\operatorname{diam} E)^{(n-1)(s-1)+\varepsilon} .
$$


The restriction becomes $1 \geq(s-1)(n-1)+\varepsilon$, which is equivalent to $s \leq 1+\frac{1-\varepsilon}{n-1}$. The range for $s$ is essentially sharp, see [6].

If $q=n$, then (1.5) reduces to the estimate

$$
\operatorname{Cap}_{p}\left(E, Q_{0}, \Omega\right) \geq C(n, s, \varepsilon)|E|^{\frac{(n-1) s+1-p+\varepsilon}{n}} .
$$

The restriction becomes $s \leq 1+\frac{p-\varepsilon}{n-1}$. Note that

$$
1+\frac{p}{n-1}>\frac{n}{n-1}+\frac{p-1}{n} .
$$

This implies that if $s<1+\frac{p}{n-1}$, then $\Omega$ is a $p$-Poincaré domain. The range for $s$ is sharp, see [11].

The estimate in Theorem 1.1 is essentially sharp in the sense that the exponent of $\mathcal{H}_{\infty}^{q}(E)$ in (1.5) cannot be made strictly smaller than $\frac{s(n-1)+1-p}{q}$; see Example 4.1 below.

Our second result shows that the requirement $q \geq s(n-1)+1-p+\varepsilon$ is essentially sharp in the sense that there exists an $s$-John domain $\Omega \subset \mathbb{R}^{n}$ such that no estimate of the form as in (1.4) holds in $\Omega$ whenever $q<\min \{s(n-1)+1-p, n\}$. This is somewhat surprising since the estimate in (1.5) does not degenerate when $q<s(n-1)+1-p$.

Theorem 1.3. Fix $1 \leq p \leq n$. There exists an $s$-John domain $\Omega \subset \mathbb{R}^{n}$ such that there is a sequence of compact sets $E_{j}$ in $\Omega$ with the following properties:

- Each $E_{j}$ is disjoint from the central Whitney cube $Q_{0}$;

- $\mathcal{H}_{\infty}^{q}\left(E_{j}\right)$ is bounded from below uniformly by a positive constant and $\operatorname{Cap}_{p}\left(E_{j}, Q_{0}, \Omega\right) \rightarrow 0$ as $j \rightarrow \infty$, whenever $q<\min \{(n-$ 1) $s+1-p, n\}$.

It would be interesting to know whether one can obtain an estimate of the form as in (1.4) when $q=(n-1) s+1-p$.

When $q<\min \left\{(n-1) s+1-p, \log _{2}\left(2^{n}-1\right)\right\}$, the $s$-John domain $\Omega$ constructed in Theorem 1.3 is in fact Gromov hyperbolic in the quasihyperbolic metric. This is very surprising, since it was proven in [4] that for all Gromov hyperbolic $s$-John domains $\Omega$, an estimate of the form as in (1.4) holds when $p=n, q=1$ and $E \subset \Omega$ is a continuum. Our example shows that one can not replace the assumption being a continuum by just being compact, and still obtain the estimate for all $s$-John domains. For definitions and examples of Gromov hyperbolic domains, we refer to the beautiful monograph [2].

\section{Preliminary Results}

For an increasing function $\tau:[0, \infty) \rightarrow[0, \infty)$ with $\tau(0)=0$, we denote by $\mathcal{H}_{\infty}^{\tau}$ the Hausdorff $\tau$-content: $\mathcal{H}_{\infty}^{\tau}(E)=\inf \sum_{i} \tau\left(r_{i}\right)$, where the infimum is taken over all coverings of $E \subset \mathbb{R}^{n}$ with balls $B\left(x_{i}, r_{i}\right)$, $i=1,2, \ldots$ When $\tau(t)=t^{s}$ for some $0<s<\infty$, we write $\mathcal{H}_{\infty}^{s}=\mathcal{H}_{\infty}^{\tau}$. 
For disjoint compact sets $E$ and $F$ in the domain $\Omega$, we denote by $\operatorname{Cap}_{p}(E, F, \Omega)$ the $p$-capacity of the pair $(E, F)$ :

$$
\operatorname{Cap}_{p}(E, F, \Omega)=\inf _{u} \int_{\Omega}|\nabla u(x)|^{p} d x
$$

where the infimum is taken over all continuous functions $u \in W_{l o c}^{1, p}(\Omega)$ which satisfy $u(x) \leq 0$ for $x \in E$ and $u(x) \geq 1$ for $x \in F$.

Let $\Omega$ be a bounded domain in $\mathbb{R}^{n}, n \geq 2$. Then $\mathbb{W}=\mathbb{W}(\Omega)$ denotes a Whitney decomposition of $\Omega$, i.e. a collection of closed cubes $Q \subset \Omega$ with pairwise disjoint interiors and having edges parallel to the coordinate axes, such that $\Omega=\cup_{Q \in \mathbb{W}} Q$, the diameters of $Q \in \mathbb{W}$ belong to the set $\left\{2^{-j}: j \in \mathbb{Z}\right\}$ and satisfy the condition

$$
\operatorname{diam}(Q) \leq \operatorname{dist}(Q, \partial \Omega) \leq 4 \operatorname{diam}(Q)
$$

For $j \in \mathbb{Z}$ we define

$$
\mathbb{W}_{j}=\left\{Q \in \mathbb{W}: \operatorname{diam}(Q)=2^{-j}\right\} .
$$

The following lemma is well-known, see for instance [15, Lemma 2.8].

Lemma 2.1. Fix $1 \leq p<\infty$. Let $B_{1}, B_{2}, \ldots$ be balls or cubes in $\mathbb{R}^{n}$, $a_{j} \geq 0$ and $\lambda>1$. Then

$$
\left\|\sum a_{j} \chi_{\lambda B_{j}}\right\|_{p} \leq C(\lambda, n, p)\left\|\sum a_{j} \chi_{B_{j}}\right\|_{p}
$$

\section{MAIN PROOFS}

Proof of Theorem 1.1. The proof is a combination of several well-known arguments; in particular [8, Proof of Theorem 9] and [11, Proof of Theorem 5.9]. For any compact set $E \subset \Omega$ such that $E \cap Q_{0}=\emptyset$, where $Q_{0}$ is the central cube that contains the John center $x_{0}$, we fix a test function $u$ for $\operatorname{Cap}_{p}\left(E, Q_{0}, \Omega\right)$, i.e. $u$ is a continuous function in $W_{l o c}^{1, p}(\Omega)$ so that $u \geq 1$ on $E$ and $u \leq 0$ on $Q_{0}$. We may assume that $\operatorname{diam} \Omega=1$.

For each $x \in E$, we may fix an $s$-John curve $\gamma$ joining $x$ to $x_{0}$ in $\Omega$ and define $P(x)$ to be the collection of Whitney cubes that intersect $\gamma$. Thus $Q(x) \in P(x)$ will be the Whitney cube containing the point $x$. We next divide our compact set $E$ into the good part and the bad part according to the range of $u_{Q}$. Let $\mathscr{G}=\left\{x \in E: u_{Q(x)} \leq \frac{1}{2}\right\}$ and $\mathscr{B}=E \backslash \mathscr{G}$.

Claim 1: for $1 \leq p \leq n$ and $q \geq s(n-1)+1-p+\varepsilon$, there exists a positive constant $C(n, p, q, s, \varepsilon)$ such that

$$
\int_{\Omega}|\nabla u(x)|^{p} d x \geq C(n, p, q, s, \varepsilon)\left(\mathcal{H}_{\infty}^{q}(\mathscr{B})\right)^{\frac{s(n-1)+1-p+\varepsilon}{q}} .
$$

Proof of Claim 1: Fix $1 \leq p \leq n, q \geq s(n-1)+1-p+\varepsilon$ and set $\Delta=\frac{\varepsilon}{2}$. Let $Q_{i}, i=1, \ldots, m$ be those Whitney cubes that intersect $\mathscr{B}$. Fix one such Whitney cube $Q_{i_{0}}$ and let $x_{i_{0}}$ be its center. Let $Q_{i_{0}}^{j}, j=1, \ldots, k$ 
be the Whitney cubes in $P\left(x_{i_{0}}\right)$ with $Q_{i_{0}}^{k}=Q_{i_{0}}$. The standard chaining argument involving Poincaré inequality [21] gives us the estimate

$$
1 \lesssim \sum_{j=1}^{k} \operatorname{diam} Q_{i_{0}}^{j} f_{Q_{i_{0}}^{j}}|\nabla u(y)| d y
$$

Hölder's inequality implies

$$
1 \lesssim\left(\sum_{j=0}^{k} r_{j}^{(1-\kappa) p /(p-1)}\right)^{(p-1) / p}\left(\sum_{j=0}^{k} r_{j}^{\kappa p-n} \int_{Q_{i_{0}}^{j}}|\nabla u|^{p}\right)^{1 / p}
$$

where $r_{j}=\operatorname{diam} Q_{i_{0}}^{j}$ and $\kappa=\frac{s+p-1-\Delta}{s p}$. Using the $s$-John condition, one can easily conclude

$$
\sum_{j=0}^{k} r_{j}^{(1-\kappa) p /(p-1)}<C
$$

Therefore,

$$
\sum_{j=0}^{k} r_{j}^{\kappa p-n} \int_{Q_{i_{0}}^{j}}|\nabla u|^{p} \geq C
$$

where the constant $C$ depends only on $p, n, \Delta$ and the constant from the $s$-John condition.

By the $s$-John condition $C r_{j} \geq|x-y|^{s}$, for $y \in Q_{i_{0}}^{j}$, and since $\kappa p-n<0$ according to our choice $p \leq n$, we obtain

$$
r_{j}^{\kappa p-n} \lesssim|x-y|^{s(\kappa p-n)}
$$

for $y \in Q_{i_{0}}^{j}$. For $y \in Q_{i_{0}}^{i} \cap\left(2^{j+1} Q_{i_{0}} \backslash 2^{j} Q_{i_{0}}\right)$, we have $|x-y| \approx 2^{j} r_{k}$ and hence for such $y$,

$$
r_{i}^{\kappa p-n} \lesssim\left(2^{j} r_{k}\right)^{s(\kappa p-n)}
$$

Combining (3.2) with (3.3) leads to

$$
\begin{aligned}
1 & \lesssim \sum_{j=0}^{k} r_{j}^{\kappa p-n} \int_{Q_{i_{0}}^{j}}|\nabla u|^{p} \lesssim\left(r_{k}\right)^{s(\kappa p-n)} \int_{Q_{i_{0}}}|\nabla u|^{p} \\
& +\sum_{j=0}^{\left|\log r_{k}\right|}\left(2^{j} r_{k}\right)^{s(\kappa p-n)} \int_{\left(2^{j+1} Q_{i_{0}} \backslash 2^{j} Q_{i_{0}}\right) \cap \Omega}|\nabla u|^{p} \\
& \lesssim \sum_{l=0}^{\left|\log r_{k}\right|+1}\left(2^{l} r_{k}\right)^{s(\kappa p-n)} \int_{2^{l} Q_{i_{0}} \cap \Omega}|\nabla u|^{p} .
\end{aligned}
$$

On the other hand,

$$
\sum_{l=0}^{\left|\log r_{k}\right|+1}\left(2^{l} r_{k}\right)^{\Delta}<r_{k}^{\Delta} \sum_{l=-\infty}^{\left|\log r_{k}\right|+1} 2^{l \Delta}<C
$$


Combining the above two estimates, we conclude that there exists an $l$ (depending on $\Delta$ and hence $\varepsilon$ ) such that

$$
\left(2^{l} r_{k}\right)^{\Delta} \lesssim\left(2^{l} r_{k}\right)^{s(\kappa p-n)} \int_{2^{l} Q_{i_{0}} \cap \Omega}|\nabla u|^{p}
$$

It follows that,

$$
\int_{\Omega \cap 2^{l} Q_{i_{0}}}|\nabla u|^{p} \gtrsim\left(2^{l} r_{k}\right)^{s(n-\kappa p)+\Delta}=\left(2^{l} r_{k}\right)^{s(n-1)+1-p+\varepsilon} .
$$

In other words, there exists an $R_{x} \geq d(x, \partial \Omega) / 2$ with

$$
\left(\int_{\Omega \cap B\left(x, R_{x}\right)}|\nabla u|^{p}\right)^{\frac{q}{s(n-1)+1-p+\varepsilon}} \gtrsim R_{x}^{q} .
$$

Applying the Vitali covering lemma to the covering $\left\{B\left(x, R_{x}\right)\right\}_{x \in E}$ of the set $\mathscr{B}$, we can select pairwise disjoint balls $B_{1}, \ldots, B_{k}, \ldots$ such that $\mathscr{B} \subset \bigcup_{i=1}^{\infty} 5 B_{i}$. Let $r_{i}$ denote the radius of the ball $B_{i}$. Then

$$
\begin{aligned}
\mathcal{H}_{\infty}^{q}(\mathscr{B}) & \leq \sum_{i=1}^{\infty}\left(\operatorname{diam} 5 B_{i}\right)^{q}=5^{q} \sum_{i=1}^{\infty} r_{i}^{q} \\
& \lesssim \sum_{i=1}^{\infty}\left(\int_{\Omega \cap B_{i}}|\nabla u|^{p}\right)^{\frac{q}{s(n-1)+1-p+\varepsilon}}
\end{aligned}
$$

The desired capacity estimate follows by noticing the elementary inequality

$$
\sum_{i} a_{i}^{b} \lesssim\left(\sum_{i} a_{i}\right)^{b}, \quad b \geq 1 .
$$

Claim 2: for $n-q<p \leq n$ and $0<\varepsilon<p+q-n$,

$$
\int_{\Omega}|\nabla u(x)|^{p} d x \geq C(p, q, n, \varepsilon)\left(\mathcal{H}_{\infty}^{q}(\mathscr{G})\right)^{\frac{n-p+\varepsilon}{q}} .
$$

Proof of Claim 2: Fix $n-q<p \leq n$ and $0<\varepsilon<p+q-n$. Our aim is to show that

$$
\int_{2 Q(x)}|\nabla u(x)|^{p} d x \geq C(p, s, n) \mathcal{H}_{\infty}^{s}(\mathscr{G} \cap Q(x))
$$

for any $n-p<s \leq n$. We adapt the argument from [11, Proof of Theorem 5.9].

Fix $n-p<s \leq n$. For $y \in \mathscr{G}, u_{Q(y)} \leq \frac{1}{2}$. For $x \in \mathscr{G} \cap Q(y)$, write $Q_{i}=Q\left(x, r_{i}\right)$, where $r_{i}=2^{-i-1} \operatorname{diam} Q(y)$. Then

$$
u(x)=\lim _{i \rightarrow \infty} u_{Q_{i}}=\lim _{i \rightarrow \infty} f_{Q_{i}} u .
$$

Now

$$
\frac{1}{2} \leq\left|u(x)-u_{Q_{0}}\right| \leq \sum_{i \geq 0}\left|u_{Q_{i}}-u_{Q_{i+1}}\right|
$$


Since by the Poincaré inequality

$$
\left|u_{Q_{i}}-u_{Q_{i+1}}\right| \leq C(n) r_{i}^{\frac{p+s-n}{p}}\left(r_{i}^{-s} \int_{Q_{i}}|\nabla u|^{p}\right)^{\frac{1}{p}}
$$

we obtain that

$$
\begin{aligned}
\frac{1}{2} & \leq \sum_{i=1}^{\infty} C(n) r_{i}^{\frac{p+s-n}{p}}\left(r_{i}^{-s} \int_{Q_{i}}|\nabla u|^{p}\right)^{\frac{1}{p}} \\
& \leq C(p, s, n)(\operatorname{diam} Q(y))^{\frac{p+s-n}{p}} \sup _{0<t \leq \operatorname{diam} Q(y)}\left(t^{-s} \int_{Q(x, t)}|\nabla u|^{p}\right)^{\frac{1}{p}} \\
& \leq C(p, s, n) \sup _{0<t \leq \operatorname{diam} Q(y)}\left(t^{-s} \int_{Q(x, t)}|\nabla u|^{p}\right)^{\frac{1}{p}} .
\end{aligned}
$$

Thus, for each $x \in \mathscr{G} \cap Q(y)$, there is a cube $Q\left(x, t_{x}\right)$ such that $t_{x} \leq$ $\operatorname{diam} Q(y)$ and that

$$
t_{x}^{s} \leq C(p, s, n) \int_{Q\left(x, t_{x}\right)}|\nabla u|^{p} .
$$

By Vitali we can find pairwise disjoint cubes $Q_{1}, Q_{2}, \ldots$ as above such that $\mathscr{G} \cap Q(y) \subset \bigcup 5 Q_{i}$. Then

$$
\begin{aligned}
\mathcal{H}_{\infty}^{s}(\mathscr{G} \cap Q(y)) & \leq C(p, s, n) \sum_{i=1}^{\infty} \int_{Q_{i}}|\nabla u|^{p} \\
& \leq C(p, s, n) \int_{2 Q(y)}|\nabla u|^{p}
\end{aligned}
$$

Thus the proof of (3.5) is complete.

We next show that for $n-q<p \leq n$ and for fixed $0<\varepsilon<p+q-n$, the following estimate holds.

$$
\int_{2 Q(x)}|\nabla u(x)|^{p} d x \geq C(p, q, n, \varepsilon)\left(\mathcal{H}_{\infty}^{q}(\mathscr{G} \cap Q(x))\right)^{\frac{n-p+\varepsilon}{q}}
$$

Let $\varepsilon>0$ be as above. We set $s=n-p+\varepsilon$. Then $s<q$. Now (3.6) follows from (3.5) and the trivial estimate

$$
\left(\mathcal{H}_{\infty}^{q}(E)\right)^{\frac{s}{q}} \lesssim \mathcal{H}_{\infty}^{s}(E)
$$

Taking into account the sub-additivity of Hausdorff $q$-content and concavity of the function $t \mapsto t^{\frac{n-p+\varepsilon}{q}}$, (3.4) follows immediately from (3.6) and Lemma 2.1. 


\section{ExAmples}

Example 4.1. We will use the standard "rooms and corridors" type domains. This type of domains consists of a central cube shaped room along with an infinite disjoint collection of cube shaped rooms which are connected to the central room by narrow cylindrical corridors; see Figure 1 .

For each $j \in \mathbb{N}$, the attached cube shaped room $E_{j}$ is of edge length $r_{j}$ and the narrow cylindrical corridor is of radius $r_{j}^{s}$ and height $r_{j}$. We can ensure that the rooms and corridors are pairwise disjoint by requiring the sequence $\left\{r_{j}\right\}_{j \in \mathbb{N}}$ to decrease to zero sufficiently rapidly. It is clear that $\Omega$ is an $s$-John domain.

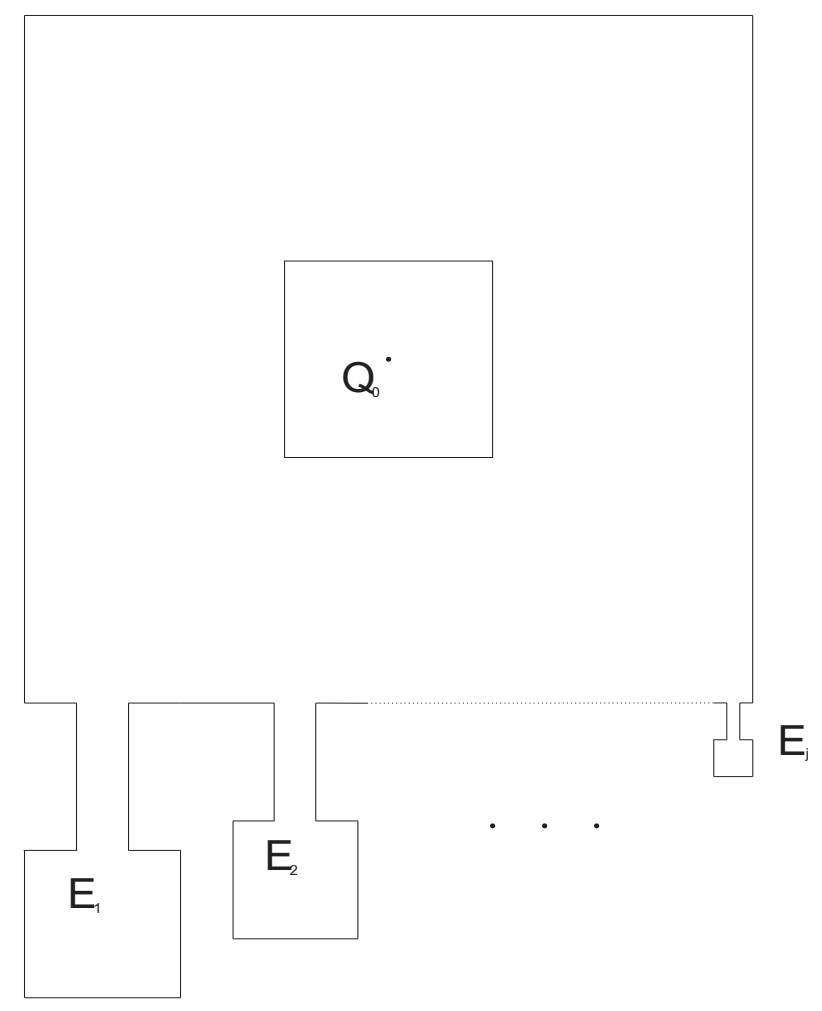

FiguRE 1. The standard "room and corridors" type domain

For $s<\frac{p+q-1}{n-1}$, we may choose $\varepsilon>0$ such that $q \geq s(n-1)+1-p+\varepsilon$. Then it is easy to obtain the following estimate:

$$
\operatorname{Cap}_{p}\left(E_{j}, Q_{0}, \Omega\right) \leq C r_{j}^{(n-1) s-p+1} \leq C \mathcal{H}_{\infty}^{q}\left(E_{j}\right)^{\frac{(n-1) s-p+1}{q}}
$$

Noticing that $r_{j} \rightarrow 0$ as $j \rightarrow \infty$, this implies that the exponent of $\mathcal{H}_{\infty}^{q}(E)$ in Theorem 1.1 is essentially best possible.

Example 4.2. Fix $p \in[1, n], n \geq 2$. There exists an $s$-John domain $\Omega$ in $\mathbb{R}^{n}$ such that there is a sequence of compact sets $E_{j}$ in $\Omega$ with the following two properties: 
- Each $E_{j}$ is disjoint from the central Whitney cube $Q_{0}$;

- $\mathcal{H}_{\infty}^{q}\left(E_{j}\right)$ is bounded from below uniformly by a positive constant and $\operatorname{Cap}_{p}\left(E_{j}, Q_{0}, \Omega\right) \rightarrow 0$ as $j \rightarrow \infty$, whenever $n-1 \leq q<$ $\min \{(n-1) s+1-p, n\}$.

The idea of the construction of such an $s$-John domain is the following: we first construct a John domain $\Omega_{0}$ such that the number $N_{j}$ of Whitney cubes of size (comparable to) $r_{j}=2^{-j}$ in $\Omega_{0}$ is approximately $2^{q j}$. We then build a "room and $s$-passage" $Q_{s}$ in each Whitney cube $Q \subset \Omega_{0}$ and $Q \neq Q_{0}$, where $Q_{0}$ is the central Whitney cube $Q_{0}$ containing the John center; see Figure 3 . If the Whitney cube $Q$ is of edge length $4 r_{j}$, then the attached room shaped cube is of side length $r_{j}$ and the corresponding $s$-passage is of radius $r_{j}^{s}$ and height $r_{j}$.

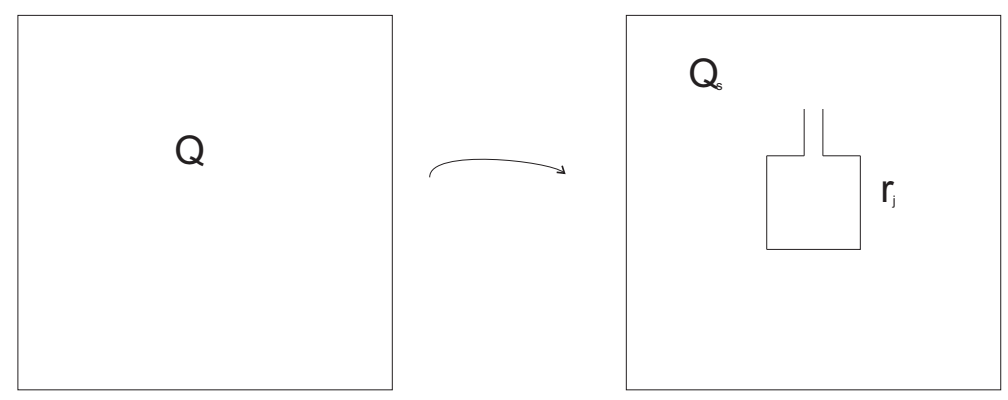

FiguRe 2. "room and s-passage" type replacement

Let $E_{j}$ be the union of all the room shaped cube of edge length $r_{j}$. Then we have the following trivial upper estimate

$$
\operatorname{Cap}_{p}\left(E_{j}, Q_{0}, \Omega\right) \leq C N_{j} \cdot r_{j}^{(n-1) s-p+1} \leq C r_{j}^{(n-1) s-p-q+1} .
$$

Thus $\operatorname{Cap}_{p}\left(E_{j}, Q_{0}, \Omega\right) \rightarrow 0$ whenever $q<(n-1) s-p+1$. On the other hand, noting that all the cubes in $E_{j}$ are well separated, to estimate the Hausdorff $q$-content, one has to cover each such cube by a ball of the same size (since otherwise the ball will intersects two cubes and substantially increases the radius). Thus we have

$$
\mathcal{H}_{\infty}^{q}\left(E_{j}\right) \geq C N_{j} \cdot r_{j}^{q} \geq C .
$$

To construct a John domain with the desired property, one essentially needs to construct a John domain $\Omega_{0}$ such that $\operatorname{dim}_{\mathcal{M}}\left(\partial \Omega_{0}\right)=q$ when $q \in[n-1, n)$, where $\operatorname{dim}_{\mathcal{M}}$ denotes the upper Minkowski dimension. With this understood, one can select certain Von Koch type curve as the boundary of a John domain; see [10, Proposition 5.2] for the detailed construction of such a John domain $\Omega_{0}$. It is clearly that the "room and $s$-passage" type replacement described above turns $\Omega_{0}$ into an $s$-John domain $\Omega$. In fact, $\operatorname{dim}_{\mathcal{M}}\left(\partial \Omega_{0}\right)=\operatorname{dim}_{\mathcal{M}}(\partial \Omega)=q$. For these facts, see [10, Proposition 5.11 and Proposition 5.16]. 
Example 4.3. Fix $1 \leq p \leq n$. There exists an $s$-John domain, which is Gromov hyperbolic in the quasihyperbolic metric, such that there is a sequence of compact sets $E_{j}$ in $\Omega$ with the follow properties:

- Each $E_{j}$ is disjoint from the central Whitney cube $Q_{0}$;

- $\mathcal{H}_{\infty}^{q}\left(E_{j}\right)$ is bounded from below uniformly by a positive constant and $\operatorname{Cap}_{p}\left(E_{j}, Q_{0}, \Omega\right) \rightarrow 0$ as $j \rightarrow \infty$, whenever $q<\min \{(n-$ 1) $\left.s+1-p, \log _{2}\left(2^{n}-1\right)\right\}$.

We first give a detailed construction of the $s$-John domain $\Omega$ in the plane with the desired properties. Fix $1 \leq p \leq 2$. We first consider the case $q=\log _{2} 3$. The $s$-John domain $\Omega$ will be constructed by an inductive process. In the first step, we have a unit cube $Q$ and four "room and $s$-passage" type "legs" as in Figure 3, The "s-passage" $R_{1}$ is a rectangle of length $2^{-1}$ and width $2^{-s-1}$ and the "room" $Q_{1}$ is a cube of edge-length $2^{-1}$. In the second step, we attach at each of the three corners of $Q_{1}$ a "room and $s$-passage" type "legs". The "s-passage" $R_{2}$ is a rectangle of length $2^{-2}$ and width $2^{-2 s-1}$ and the "room" $Q_{2}$ is a cube of edge-length $2^{-2}$. In general at step $j$, we have $4 \cdot 3^{j-1}$ "room and $s$-passage" type "legs", where the " $s$-passage" $R_{j}$ is a rectangle of length $2^{-j}$ and width $2^{-j s-1}$ and the "room" $Q_{j}$ is a cube of edge-length $2^{-j}$. It is easy to check that, with our choices of parameters, there is no overlap in our construction. Moreover, $\Omega$ is an $s$-John domain that is Gromov hyperbolic in the quasihyperbolic metric (since $\Omega$ is simply connected).

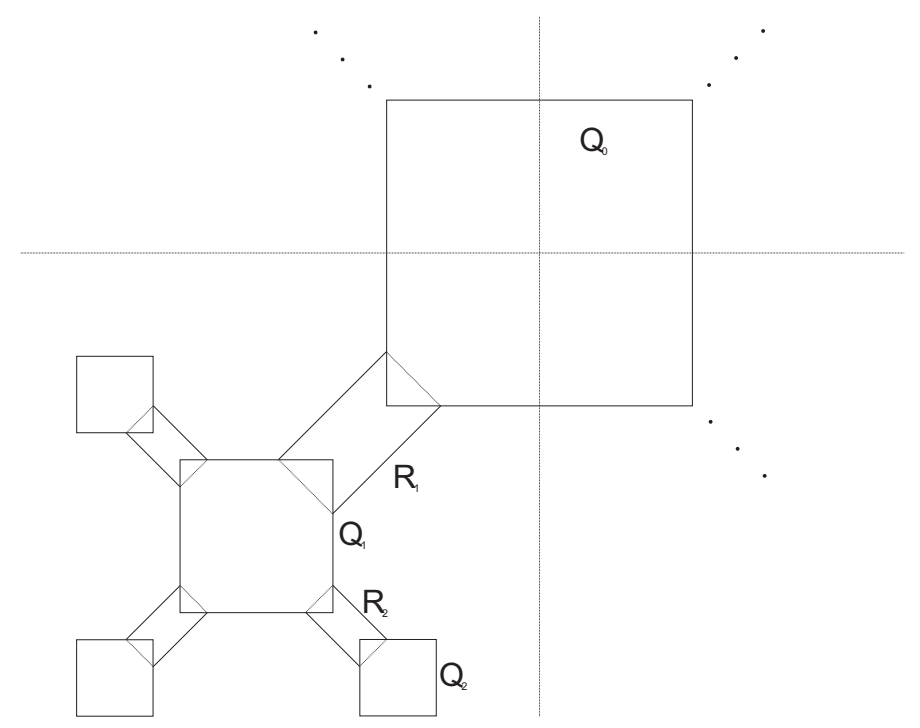

FiguRE 3 . The $s$-John domain $\Omega \subset \mathbb{R}^{2}$

We choose $E_{j}$ to be the union of all the cubes at step $j$, i.e. the collection of $4 \cdot 3^{j-1}$ (disjoint) cubes of edge-length $2^{-j}$. Noting that all the cubes at step $j$ are well separated, to estimate the Hausdorff $q$-content, one has to cover each such cube by a ball of the same size 
(since otherwise the ball will intersects two cubes and substantially increases the radius). Note also that $q=\log _{2} 3$ and so it follows that

$$
\mathcal{H}_{\infty}^{q}\left(E_{j}\right) \geq C 4 \cdot 3^{j-1} \cdot 2^{-q j}=C .
$$

On the other hand,

$$
\operatorname{Cap}_{p}\left(E_{j}, Q_{0}, \Omega\right) \leq C 4 \cdot 3^{j-1} \cdot 2^{-j(s-p+1)} \leq C 2^{-j(s-p-q+1)} .
$$

If $q<s-p+1$, then $\operatorname{Cap}_{p}\left(E_{j}, Q_{0}, \Omega\right) \rightarrow 0$ as $j \rightarrow \infty$ as desired.

Next we consider the case $q<\min \left\{s-p+1, \log _{2} 3\right\}$. This case is easier and we only need to delete some "room and $s$-passage" type "legs" from the previous construction. To be more precise, we choose $k_{j} \in \mathbb{N}$ to be an integer such that $k_{j}-1 \leq 2^{q j} \leq k_{j}$. The construction of the desired $s$-John domain can be proceeded in a similar way. In the first step, we have a unit cube $Q$ and $k_{1}$ "room and $s$-passage" type "legs" as in the previous construction. The "s-passage" $R_{1}$ is a rectangle of length $2^{-1}$ and width $2^{-s-1}$ and the "room" $Q_{1}$ is a cube of edge-length $2^{-1}$. In the second step, we fix $k_{2}$ corners of all the cubes of edge-length $2^{-1}$ in step 1, and attach at each corner a "room and $s$-passage" type "legs". The "s-passage" $R_{2}$ is a rectangle of length $2^{-2}$ and width $2^{-2 s-1}$ and the "room" $Q_{2}$ is a cube of edge-length $2^{-2}$. In general at step $j$, we have $k_{j}$ "room and $s$-passage" type "legs", where the "s-passage" $R_{j}$ is a rectangle of length $2^{-j}$ and width $2^{-j s-1}$ and the "room" $Q_{j}$ is a cube of edge-length $2^{-j}$.

Let $E_{j}$ be the union of all the cubes at step $j$, i.e. the collection of $k_{j}$ (disjoint) cubes of edge-length $2^{-j}$. It is clear that

$$
\mathcal{H}_{\infty}^{q}\left(E_{j}\right) \geq C k_{j} \cdot 2^{-q j} \geq C
$$

On the other hand, we have

$$
\operatorname{Cap}_{p}\left(E_{j}, Q_{0}, \Omega\right) \leq C k_{j} \cdot 2^{-j(s-p+1)} \leq C 2^{-j(s-p-q+1)} .
$$

If $q<s-p+1$, then $\operatorname{Cap}_{p}\left(E_{j}, Q_{0}, \Omega\right) \rightarrow 0$ as $j \rightarrow \infty$ as desired.

We can construct similar examples in $\mathbb{R}^{n}, n \geq 3$. Fix $1 \leq p \leq n$. Consider the difficult case $q=\log _{2}\left(2^{n}-1\right)$. The $s$-John domain $\Omega$ will be constructed in a similar manner as before. In the first step, we have a unit cube $Q$ and $2^{n}$ "room and $s$-passage" type "legs". The " $s$ passage" $R_{1}$ is a cylinder of height $2^{-1}$ and radius $2^{-s-1}$ and the "room" $Q_{1}$ is a cube of edge-length $2^{-1}$. In the second step, we attach at each of the $2^{n}-1$ corners of $Q_{1}$ a "room and $s$-passage" type "legs". The "s-passage" $R_{2}$ is a cylinder of height $2^{-2}$ and radius $2^{-2 s-1}$ and the "room" $Q_{2}$ is a cube of edge-length $2^{-2}$. In general at step $j$, we have $2^{n} \cdot\left(2^{n}-1\right)^{j-1}$ "room and $s$-passage" type "legs", where the " $s$-passage" $R_{j}$ is a cylinder of height $2^{-j}$ and radius $2^{-j s-1}$ and the "room" $Q_{j}$ is a cube of edge-length $2^{-j}$. It is easy to check that, with our choices of parameters, there is no overlap in our construction. Moreover, $\Omega$ is an 
$s$-John domain that is Gromov hyperbolic in the quasihyperbolic metric. Indeed, one can easily verify that every quasihyperbolic geodesic triangle in $\Omega$ is $\delta$-thin for some $\delta<\infty$.

We choose $E_{j}$ to be the union of all the cubes at step $j$, i.e. the collection of $2^{n} \cdot\left(2^{n}-1\right)^{j-1}$ (disjoint) cubes of edge-length $2^{-j}$. Note that $q=\log _{2}\left(2^{n}-1\right)$ and we obtain that

$$
\mathcal{H}_{\infty}^{q}\left(E_{j}\right) \geq C 2^{n} \cdot\left(2^{n}-1\right)^{j-1} \cdot 2^{-q j}=C .
$$

On the other hand,

$$
\begin{aligned}
\operatorname{Cap}_{p}\left(E_{j}, Q_{0}, \Omega\right) & \leq C 2^{n} \cdot\left(2^{n}-1\right)^{j-1} \cdot 2^{-j[(n-1) s-p+1]} \\
& \leq C 2^{-j[(n-1) s-p-q+1]} .
\end{aligned}
$$

If $q<(n-1) s-p+1$, then $\operatorname{Cap}_{p}\left(E_{j}, Q_{0}, \Omega\right) \rightarrow 0$ as $j \rightarrow \infty$ as desired.

The case $q<\log _{2}\left(2^{n}-1\right)$ can be proceeded as in the planar case by deleting the extra number of "room and s-passage" type "legs" and we leave the simple verification to the interested readers.

\section{Acknowledgements}

The author would like to thank his supervisor Academy Professor Pekka Koskela for posing this question and for helpful discussions.

\section{REFERENCES}

[1] K.Astala, T.Iwaniec and G.Martin, Elliptic partial differential equations and quasiconformal mappings in the plane, Princeton University Press, Princeton, NJ, 2009.

[2] M.Bonk, J.Heinonen and P.Koskela, Uniformizing Gromov hyperbolic spaces, Astérisque No. 270 (2001), viii+99 pp.

[3] S.Buckley and P.Koskela, Sobolev-Poincaré implies John, Math. Res. Lett. 2 (1995), no. 5, 577-593.

[4] C.Y.Guo, Uniform continuity of quasiconformal mappings onto generalized John domains, Ann. Acad. Sci. Fenn. Math. (to appear)

[5] C.Y.Guo, Generalized quasidisks and conformality II, Proc.Amer.Math.Soc. (to appear)

[6] C.Y.Guo and P.Koskela, Sharpness of uniform continuity of quasiconformal mappings onto $s$-John domains, submitted 2013.

[7] C.Y.Guo, P.Koskela and J.Takkinen, Generalized quasidisks and conformality, Publ.Mat., 58 (2014), no. 1, 193-212.

[8] P.Hajlasz and P.Koskela, Isoperimetric inequalities and imbedding theorems in irregular domains, J. London Math. Soc. (2) 58 (1998), no. 2, 425-450.

[9] P.Hajlasz and P.Koskela, Sobolev met Poincaré, Mem. Amer. Math. Soc. 145 (2000), no. 688 .

[10] P.Harjulehto, R.Hurri-Syrjänen, and A.V.Vähäkangas, On the $(1, p)$-Poincaré inequality, Illinois J.Math. (to appear).

[11] J.Heinonen and P.Koskela, Quasiconformal maps in metric spaces with controlled geometry, Acta Math. 181 (1998), 1-61.

[12] S.Hencl and P.Koskela, Quasihyperbolic boundary conditions and capacity: uniform continuity of quasiconformal mappings, J. Anal. Math. 96 (2005), 1935 .

[13] F.John, Rotation and strain, Comm. Pure Appl. Math. 14(1961), 391-413. 
[14] T.Kilpeläinen and J.Malý, Sobolev inequalities on sets with irregular boundaries, Z. Anal. Anwendungen 19 (2000), no. 2, 369-380.

[15] P.Koskela, Lectures on quasiconformal and quasisymmetric mappings, Jyv.Lect.Math.1, in press.

[16] P.Koskela, J.Onninen and J.T.Tyson, Quasihyperbolic boundary conditions and capacity: Hölder continuity of quasiconformal mappings, Comment. Math. Helv. 76 (2001), no. 3, 416-435.

[17] P.Koskela, J.Onninen and J.T.Tyson, Quasihyperbolic boundary conditions and Poincaré domains, Math. Ann. 323 (2002), no. 4, 811-830.

[18] O.Martio and J.Sarvas, Injectivity theorems in plane and space, Ann. Acad. Sci. Fenn. Ser. A I Math. 4 (1979), no. 2, 383-401.

[19] V.G.Maz'ya, Classes of domains and imbedding theorems for function spaces, Dokl. Akad. Nauk SSSR 133 527-530 (Russian); translated as Soviet Math. Dokl. 1 (1960) 882-885.

[20] V.G.Maz'ya, Sobolev spaces with applications to elliptic partial differential equations, Second, revised and augmented edition, Grundlehren der Mathematischen Wissenschaften [Fundamental Principles of Mathematical Sciences], 342. Springer, Heidelberg, 2011. xxviii+866 pp.

[21] W.Smith and D.A.Stegenga, Hölder and Poincaré domains, Trans. Amer. Math. Soc. 319 (1990), 67-100.

(Chang-Yu Guo) Department of Mathematics and Statistics, UniverSity of JyvÄskylä, P.O. Box 35, FI-40014 University of JyvëSKYlÄ, FinLAND

E-mail address: changyu.c.guo@jyu.fi 